\title{
Impurity Role in Mechanically Induced Defects
}

R.H. Howell, P. Asoka-Kumar, J. Hartley and P. Sterne

\section{February 25, 2000}

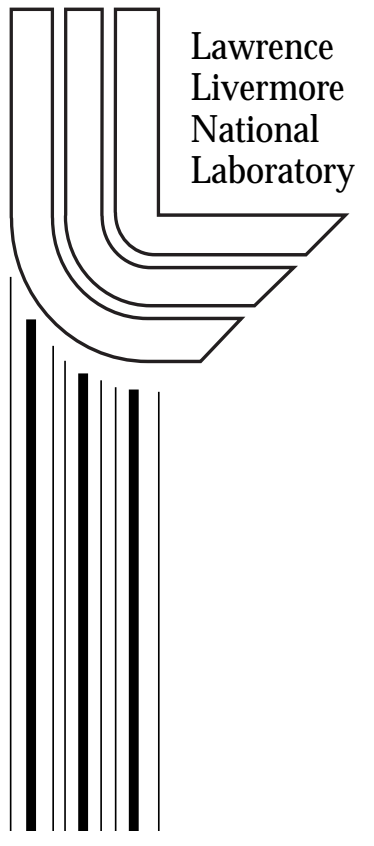




\section{DISCLAIMER}

This document was prepared as an account of work sponsored by an agency of the United States Government. Neither the United States Government nor the University of California nor any of their employees, makes any warranty, express or implied, or assumes any legal liability or responsibility for the accuracy, completeness, or usefulness of any information, apparatus, product, or process disclosed, or represents that its use would not infringe privately owned rights. Reference herein to any specific commercial product, process, or service by trade name, trademark, manufacturer, or otherwise, does not necessarily constitute or imply its endorsement, recommendation, or favoring by the United States Government or the University of California. The views and opinions of authors expressed herein do not necessarily state or reflect those of the United States Government or the University of California, and shall not be used for advertising or product endorsement purposes.

Work performed under the auspices of the U. S. Department of Energy by the University of California Lawrence Livermore National Laboratory under Contract W-7405-Eng-48.

This report has been reproduced directly from the best available copy.

Available to DOE and DOE contractors from the

Office of Scientific and Technical Information

P.O. Box 62, Oak Ridge, TN 37831

Prices available from (423) 576-8401

http://apollo.osti.gov/bridge/

Available to the public from the National Technical Information Service

U.S. Department of Commerce 5285 Port Royal Rd., Springfield, VA 22161 http://www.ntis.gov/

OR

Lawrence Livermore National Laboratory Technical Information Department's Digital Library http://www.llnl.gov/tid/Library.html 


\section{LDRD Final Report: \\ Impurity role in mechanically induced defects. \\ R. H. Howell, P. Asoka-Kumar, J. Hartley, P. Sterne \\ Lawrence Livermore National Laboratory \\ Livermore CA 94550}

An improved understanding of dislocation dynamics and interactions is an outstanding problem in the multi scale modeling of materials properties, and is the current focus of major theoretical efforts world wide. We have developed experimental and theoretical tools that will enable us to measure and calculate quantities defined by the defect structure. Unique to the measurements is a new spectroscopy that determines the detailed elemental composition at the defect site.

The measurements are based on positron annihilation spectroscopy performed with a $3 \mathrm{MeV}$ positron beam [1 ]. Positron annihilation spectroscopy is highly sensitive to dislocations and associated defects and can provide unique elements of the defect size and structure. Performing this spectroscopy with a highly penetrating positron beam enables flexibility in sample handling. Experiments on fatigued and stressed samples have been done and insitu measurement capabilities have been developed. We have recently performed significant upgrades to the accelerator operation and novel new experiments have been performed [2-4 ]

To relate the spectrographic results and the detailed structure of a defect requires detailed calculations. Measurements are coupled with calculated results based on a description of positions of atoms at the defect. This gives an atomistic view of dislocations and associated defects including impurity interactions. Our ability to probe impurity interactions is a unique contribution to defect understanding not easily addressed by other atomistic spectroscopies.

We have expanded our positron spectroscopic capabilities using the high energy positron beam to include both positron lifetime spectroscopy to determine general defect structure and concentration 
and orbital electron momentum spectroscopy to complement the lifetime spectroscopy and to determine the elemental distribution at the defect[5 ]. Using these spectroscopies we have completed two experiments in metals that clearly identify elemental impurity interactions with defects. Coupling with corresponding theoretical calculations provides a fully analytical tool.

We measured a catalog of pure elements to establish an experimental orbital electron momentum data base and validate the theoretical models. Fig. 1 shows the electron momentum distribution plotted as a function the longitudinal momentum $\left(\mathrm{p}_{\mathrm{L}}\right)$ of the electronpositron pair. The longitudinal momentum represents the momentum of the electron-positron pair prior to the annihilation. Since the positron is thermalized, the momentum of the electron-positron pair is dominated by contributions from electrons. Higher momentum components reveal the structure of the orbital electrons leading to different peak position, amplitude, and shape for different elements. The shape variations arise due to the different momentum distributions of the orbital electrons. Because the orbital electrons retain their atomic character even when atoms form a solid, these results can be directly compared to simple theoretical predictions.

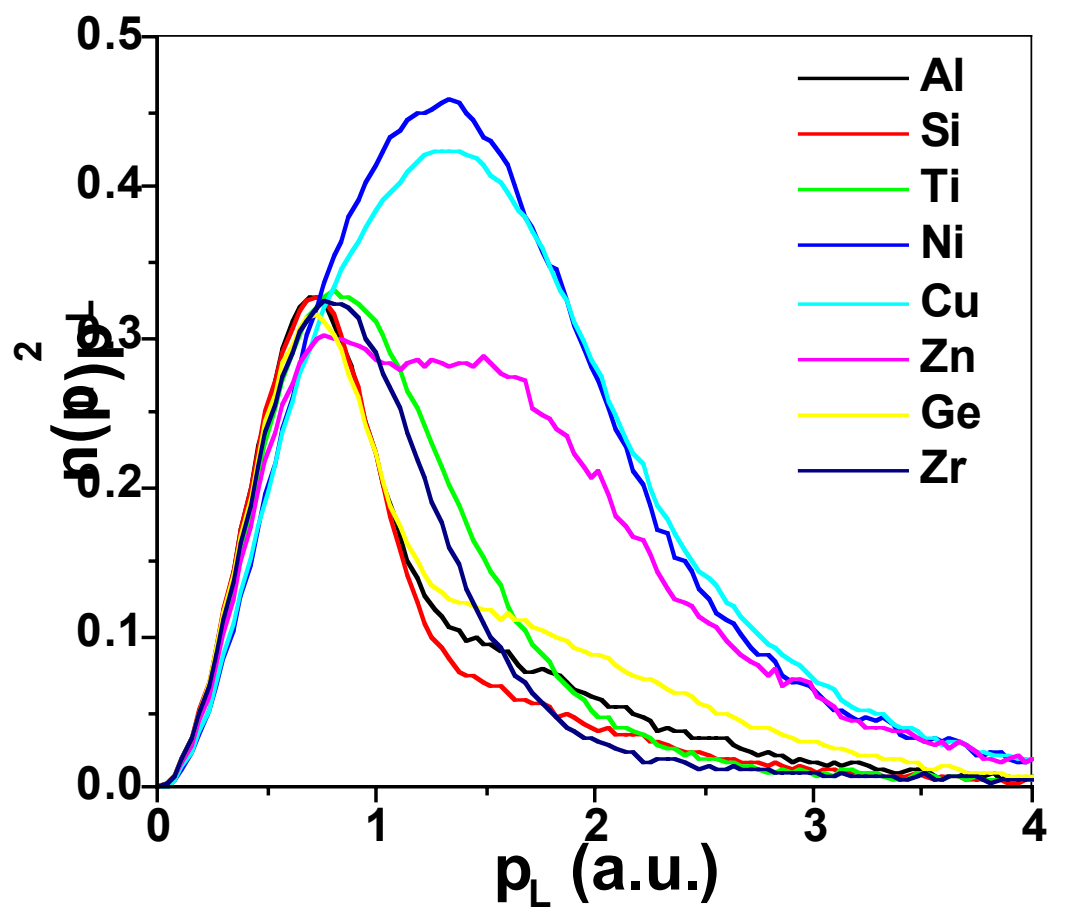

Figure 1. High values of electron momenta reflect elemental differences of orbital electrons 
This system was then tested by measuring the elements surrounding the open volume in a five component $(\mathrm{Zr}, \mathrm{Ti}, \mathrm{Al}, \mathrm{Ni}, \mathrm{Cu}$ ) amorphous metallic alloy. For each state of the metallic alloy the sample data were described by a unique linear combination of the reference spectra. Fig. 2 shows the composition of the constituents obtained from the orbital electron momentum analysis. From this analysis we were able to provide the first evidence that the distribution of the elements around the open volume was highly non-uniform and strongly favored $\mathrm{Zr}$ and $\mathrm{Ti}$.

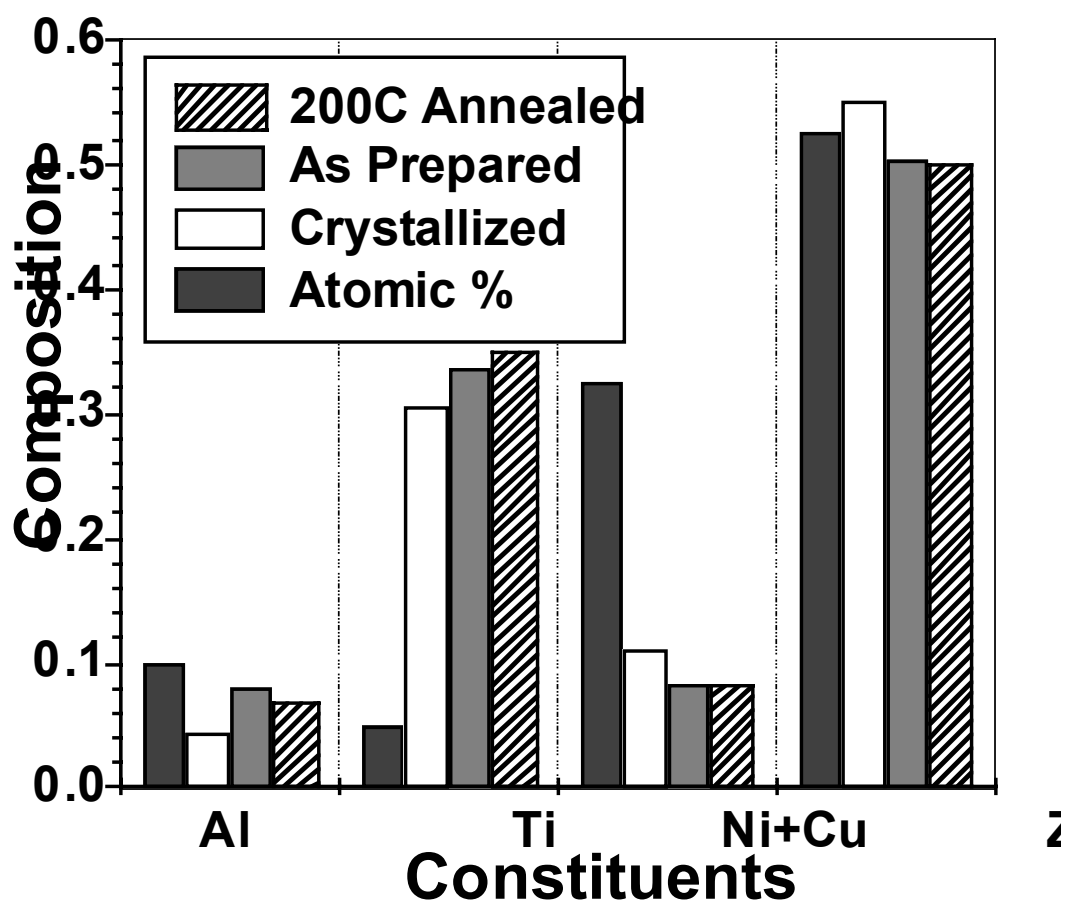

Figure 2. Non statistical distributions of elements are found around open space in complex metallic glasses

We have completed experimental and theoretical analysis of a series of stainless steel samples fatigued in small steps up to total failure and for the first time directly observed a long suspected correlation between carbon and fatigue induced defects. Positron lifetime data from the samples established the growth of two defect types. Analysis results from positron lifetime spectra are shown in Fig. 3. The top panels show the lifetime values and the bottom panels show the intensity of the second lifetime 


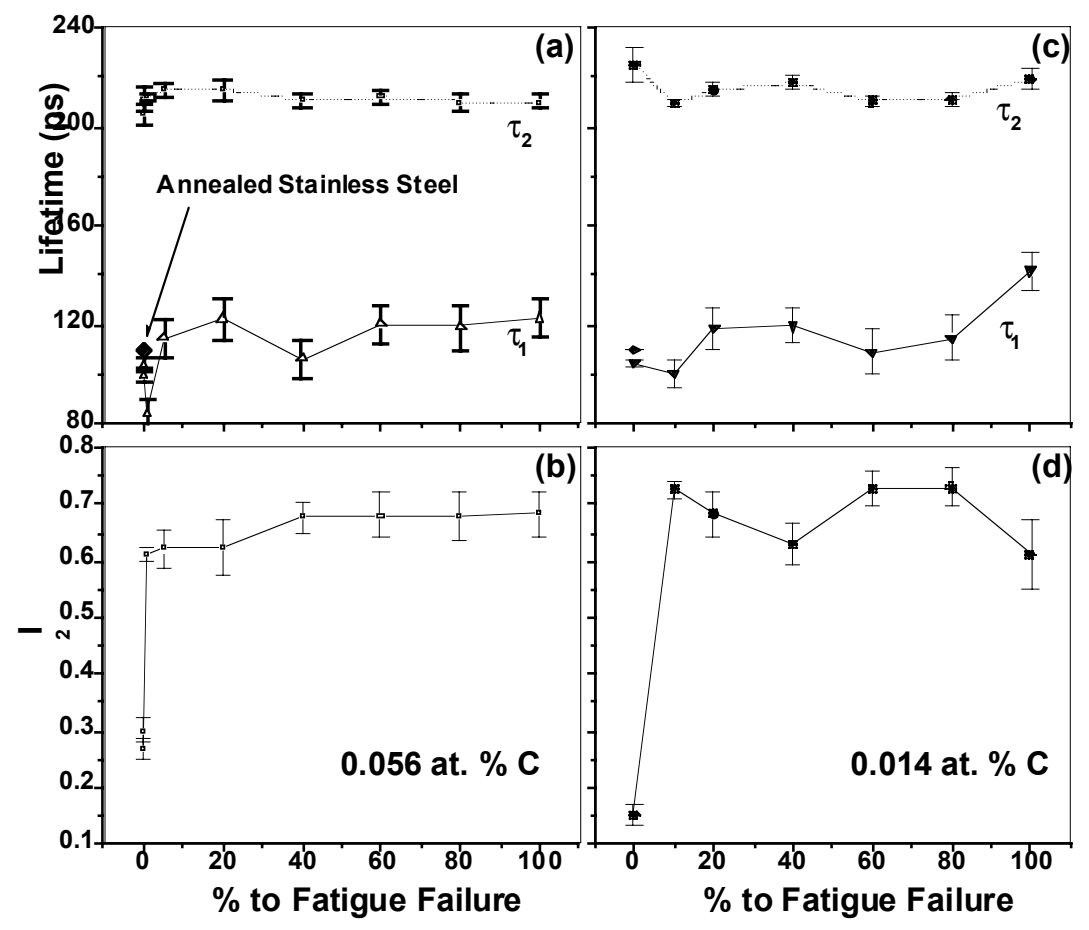

Figure 3. Positron lifetime analysis show a rapidly developed defect population during fatigue of stainless steel.

component. One defect lifetime was typical of vacancy clusters in iron and trapped $80 \%$ of the positrons at high fatigue levels. The second trap lifetime was typical of dislocation cores or grain boundaries. Orbital electron momentum measurements showed a combination of carbon and steel that matched the trapping fraction of the vacancy cluster defect. Fig. 4 shows the electron momentum distribution of as-prepared and fatigued stainless steel samples. Samples are fatigued to $1 \%-100 \%$ failure. The annealed stainless steel and graphite (carbon) data are well separated with distinct shapes. Results from as-prepared and fatigued samples fall into two distinct groups, both showing a shift towards the graphite spectrum. The shift indicates that the local environment of the positron trap 


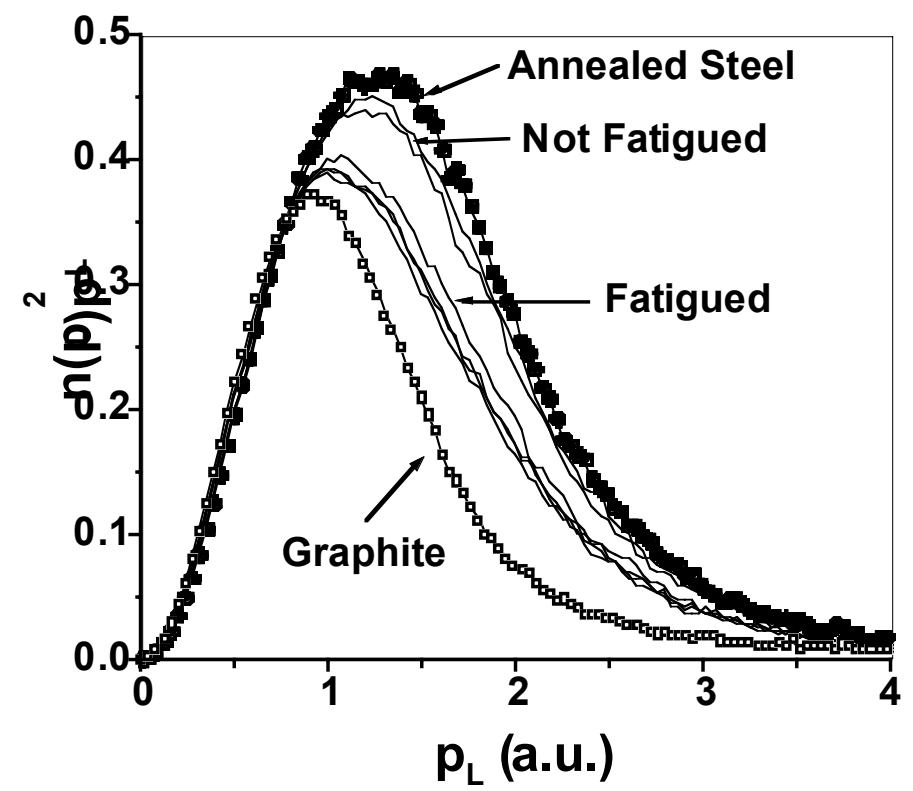

Figure 4. Linear combinations of defect free stainless steel describe the fatigued state and directly demonstrate carbon decoration of the defects.

site is strongly carbon decorated. Our calculations show that vacancy cluster carbon decoration must be nearly complete to achieve the observed combination of carbon fraction and lifetime. Fig. 5 shows the positron annihilation fraction with low momentum electrons versus high momentum electrons. When plotted in this manner, a single type of defect with varying concentrations will follow a single straight line. Data indicates a new defect generated under fatigue with a different slope consistent with positron lifetime results. 


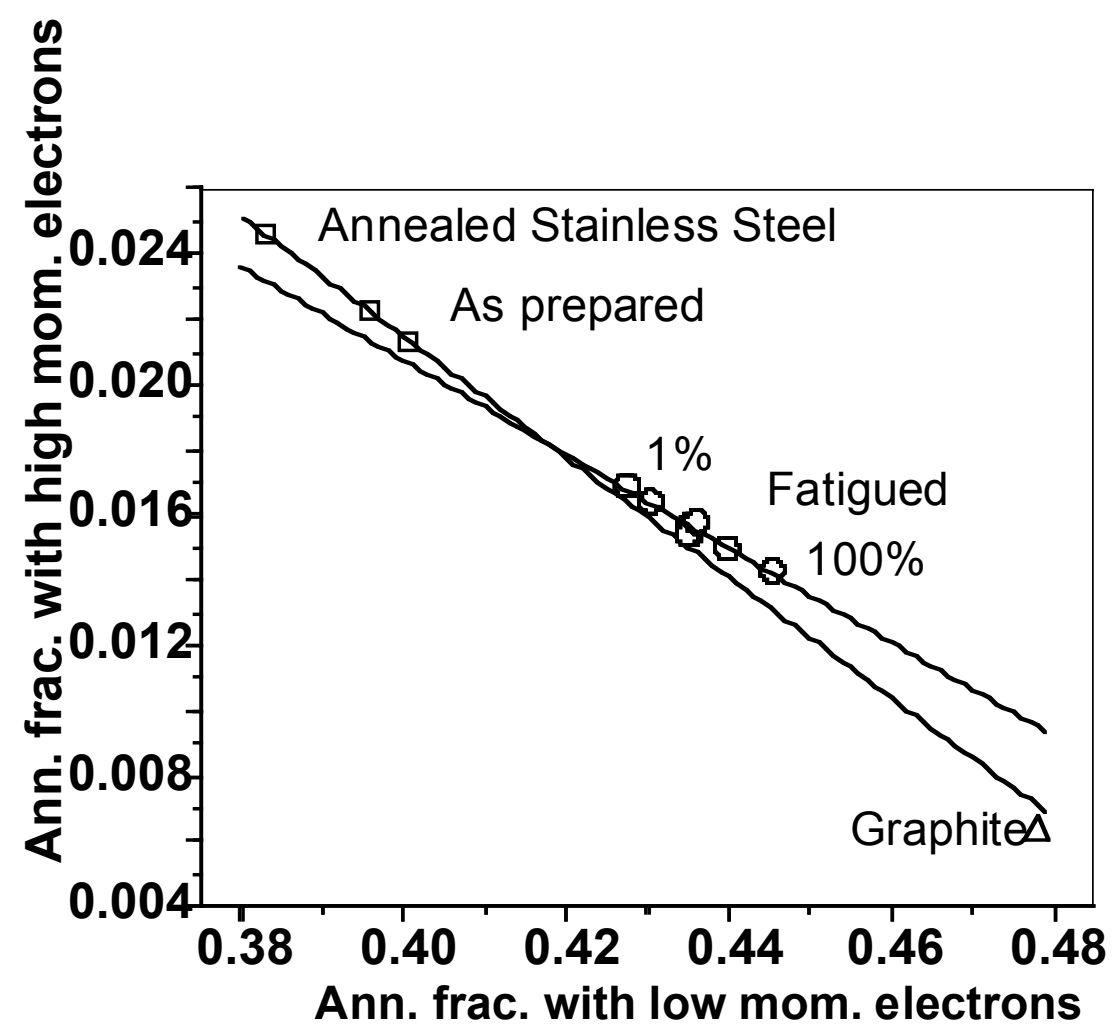

Figure 5. Comparison of the high and low momentum fractions of the electron momentum distribution demonstrate two defect states.

We have developed new calculational methods to predict positron behavior in complex defect geometries. Using a novel finite element-based electronic structure program [6], we have calculated positron distributions and lifetimes for systems ranging in size from one atom per unit cell to 2016 atoms [7]. Even larger systems are possible, and calculations have recently been performed on systems of more than 4000 atoms. This program addresses our need for a method that can calculate positron properties for realistic extended defects in materials.

To demonstrate the capabilities of this program, we have calculated positron lifetimes and distributions for a range of vacancy-related defects in elemental copper and silver. Vacancy clusters in these materials initially form three-dimensional clusters that rapidly collapse into two-dimensional Frank loops. These Frank loops, in turn, are unstable to the formation of stacking fault tetrahedra. These two vacancy cluster types form relatively extended defects in close-packed materials and bridge the gap 
between very localized defects such as monovacancies and fully extended defects like dislocations. We have calculated positron characteristics in both Frank loops and stacking fault tetrahedra for 10 and 28 vacancies in a cluster of more than 1000 atoms, as well as relaxed monovacancy structures and bulk, defect-free material. The atomic positions were extracted from larger molecular dynamics simulations using EAM potentials that were performed as part of the Enhanced Surveillance Campaign on $\mathrm{Pu}$ aging.

The calculations demonstrated the accuracy of the method, with the calculated lifetimes for defect-free copper, monovacancies, stacking fault tetrahedra and Frank loops all in very close agreement with reported experimental lifetimes values for $\mathrm{Cu}$. There were significant differences in the positron characteristics in Frank loops and stacking fault tetrahedra. Figure 6 shows the positron distribution associated with a Frank loop. This distribution is strongly localized in the region of the loop and has a relatively long lifetime of about 200ps, reflecting the open vacancy-like nature of the defect. Figure 7 shows the positron distribution around the stacking fault tetrahedron. This is much more extended in space, and in fact the positron is only weakly attracted to the defect. The more extended nature of the positron distribution is also reflected in the calculated lifetime of $123 \mathrm{ps}$, very close to the bulk defect-free lifetime value of $110 \mathrm{ps}$.

Stacking fault tetrahedra form readily in the noble metals copper, silver, and gold. They may even be present in significant quantity in well-annealed crystals. This offers an explanation for the range in published values for the positron lifetimes in well-annealed copper, which vary from $110 \mathrm{ps}$ to $125 \mathrm{ps}$. Different anneal schedules and grain sizes can all lead to different concentrations of stacking fault tetrahedra in the resulting "well-annealed" samples. Stacking fault tetrahedra and Frank loops are also relatively extended defects that display many of the characteristics associated with dislocations. Our success in these calculations therefore demonstrates the ability of this technique to treat extended defects and underlines the potential importance of positron techniques in measuring dislocation densities of interest to the LLNL Multiscale Modeling program.

We have also developed a fast and convenient atomic-based code for calculating orbital electron momentum spectra. This code, which computes the one-dimensional momentum distribution on an orbitalby-orbital basis, has been validated against experiment for a number 
of reference systems and is now used to analyze experimental data for which reference systems are unavailable.

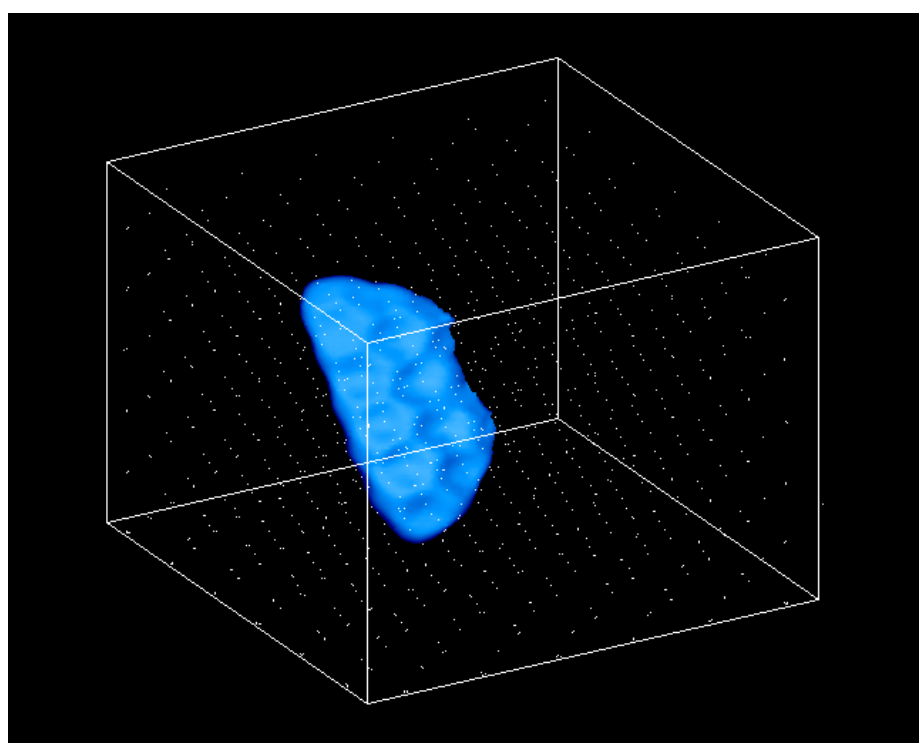

Figure 6. Positron distribution isosurface around a Frank loop in copper. The positron is strongly localized in the Frank loop and the resulting lifetime of $210 \mathrm{ps}$ is considerably longer than both the defect-free and stacking-fault-tetrahedron lifetimes. Points indicate atomic positions.

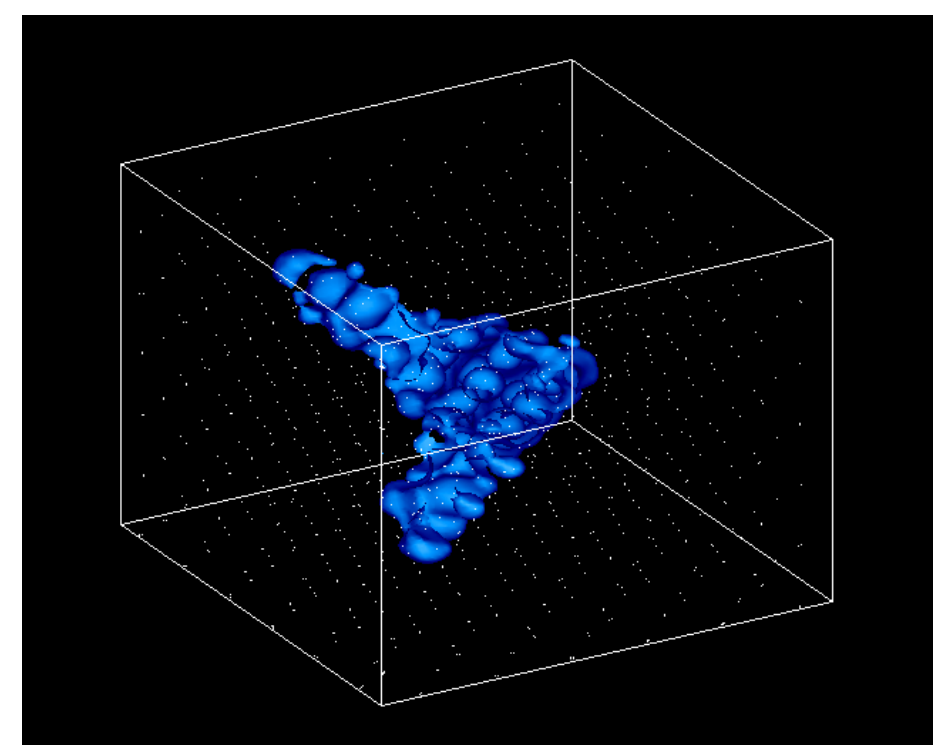

Figure 7. Positron distribution isosurface around a stacking fault tetrahedron in copper. The positron is relatively delocalized and the resulting lifetime of $122 \mathrm{ps}$ is only slightly longer than the bulk defect-free lifetime of 110 ps. Points indicate atomic positions. 
1. R. H. Howell, P. A. Sterne, J. Hartley, and T. E. Cowan "High Energy Beam Lifetime Analysis" Journal of Applied Surface Science 149, 103 (1999).

2. A.W. Hunt, D.B. Cassidy, F.A. Selim, R. Haakenaasen, T.E. Cowan, R.H. Howell, K.G. Lynn and J.A. Golovchenko, "In-flight Annihilation of Fast Positrons: Spatial Sampling of Crystal Electrons", Nature 402, 157 (1999).

3. F. A. Selim ${ }^{1,2}$, A. W. Hunt ${ }^{1,3}$, R. Howell ${ }^{1}$, K.G. Lynn ${ }^{4}$ and J. A. Golovchenko ${ }^{3,5}$ "Multiple Scattering Measurements of Energetic Positrons in a Thin Gold Polycrystal" , To be published in Nuclear Instruments and Methods B.

4. F. A. Selim, A. W. Hunt, J. A. Golovchenko, R. Howell, R.Haakenaasen, K.G. Lynn, and L. V. Hau "Improved Source and Transport of Monoenergetic MeV Positrons", To be published in Nuclear Instruments and Methods B

5. P. Asoka-Kumar, M. Alatalo, V.J. Ghosh, A.C. Kruseman, B. Nielsen and K. G. Lynn, Phys. Rev. Let. 77, 2097 (1996)

6. J.E. Pask, B.M. Klein, C.Y. Fong, and P.A. Sterne, "Real-Space Local Polynomial Basis For Solid-State Electronic-Structure Calculations: A Finite-Element Approach", Physical Review B 59, 12352 (1999).

7. P.A. Sterne, J.E. Pask, and B.M. Klein, "Calculation Of Positron Observables Using A Finite-Element-Based Approach", Applied Surface Science 149, 238 (1999). 